\title{
Elastic fields of stationary and moving dislocations in finite samples
}

\author{
Rodrigo Arias \\ Departamento de Física, Facultad de Ciencias Físicas y Matemáticas \\ Universidad de Chile, Casilla 487-3, Santiago, Chile
}

February 11, 2017

\begin{abstract}
Integral expressions are determined for the elastic displacement and stress fields due to stationary or moving dislocation loops in finite samples. These general expressions are valid for anisotropic media as well. Specifically for the stress fields, a line integral representation is found, thus showing rigorously the independence of the stress fields with respect to the choice of slip planes. In the stationary case the line integral representation involves calculating a "vector potential" dependent on the specific geometry of the sample. Two examples of geometries, isotropic half space and thin plate, are shown where the "vector potential" has been explicitly determined. With this general method one recovers some earlier specific results in these geometries.
\end{abstract}

\section{Introduction}

A general formula for the displacement and stress fields generated by a dislocation loop undergoing arbitrary motion in an infinite medium was obtained some years ago by Mura (1963). It is written as a convolution of the medium's impulse response with a source localized along the dislocation loop (i.e. independent of the loop's slip plane). These formulae do not apply to the case of finite samples since they are derived assuming homogeneity in space, i.e. a dependence of the Green's function in $\vec{x}-\vec{x}^{\prime}$, which indeed it is no longer true in a finite sample. The purpose of this paper is to announce a generalization of these formulae to finite samples, as well as explicit forms for the geometries of a half space and thin plate. The main results will be presented, with some examples, but the detailed proofs will be presented elsewhere.

Motivation for this work comes partly from an ongoing project that attempts to understand recently observed dynamic instabilities for cracks in thin plates (Sharon, Gross and Fineberg 1996, Boudet, Ciliberto and Steinberg 1996) in terms of the interaction of the plate's oscillations with the crack's tip regarded as a continuous distribution of infinitesimally small dislocations (Lund 1996). An intuitive thought behind this work was that the stress fields produced by stationary or moving dislocation loops in a finite sample should not depend on the slip planes chosen, and that indeed the stresses will be continuous through the slip planes, as it was in an infinite medium. This is rigorously shown here: the expressions found for the stress fields involve only line integrals over the dislocation loops.

When the loops are stationary in order to find the explicit form of the line integrals appearing in the stress fields, one should calculate a "vector potential" appropriate to the finite sample in 
question. This was done for the two examples shown here: a half space and a thin plate. In a non stationary situation the displacement velocity field can be written in terms of line integrals, and from this one deduces that the temporal partial derivative of the stress field can be written in terms of them too. One finally deduces that the time dependent stress field can be written in terms of line integrals since one knows that the initial condition (stationary case) and its temporal derivative can both be written in terms of line integrals.

\section{Displacement fields due to dislocations in finite samples}

\subsection{Stationary case}

The following formula is valid in a finite sample for the displacement field produced by a stationary dislocation loop (derived in detail elsewhere). It is written as an integration over the slip plane $S^{\prime}$, and involves a discontinuity of the displacement there, given by the Burgers vector $b_{i}$ :

$$
U_{m}(x)=-b_{i} \int_{S^{\prime}} d S_{j}^{\prime} \sigma_{i j}^{G m}\left(x^{\prime}, x\right) .
$$

$\sigma_{i j}^{G m}$ is the elastic stress associated with the static Green's function $G_{l m}\left(x, x^{\prime}\right)$, which is the displacement (direction $(l)$ ) produced by a localized impulse force in space $\left(x^{\prime}\right)$ (direction $(m)$ ):

$$
C_{i j k l} \frac{\partial^{2}}{\partial x_{k} \partial x_{j}} G_{l m}\left(x, x^{\prime}\right) \equiv \frac{\partial}{\partial x_{j}} \sigma_{i j}^{G m}\left(x, x^{\prime}\right)=-\delta_{i m} \delta\left(x-x^{\prime}\right) .
$$

It satisfies the free surface boundary condition:

$$
\sigma_{i j}^{G m}\left(x_{S}, x^{\prime}\right) n_{j}\left(x_{S}\right)=0,
$$

with $n_{j}\left(x_{S}\right)$ the components of the normal to the free surface at its point $x_{S}$.

\subsection{Non stationary case}

The following formula is valid in a finite sample for the displacement field produced by a moving dislocation loop. It involves integration over past slip planes $S\left(t^{\prime}\right)$ :

$$
U_{m}(x, t)=-b_{i} \int_{-\infty}^{\infty} d t^{\prime} \int_{S\left(t^{\prime}\right)} d S_{j}^{\prime} \sigma_{i j}^{G m}\left(x^{\prime}, x ; t-t^{\prime}\right) .
$$

The Green's function $G_{i m}\left(x, x^{\prime} ; t-t^{\prime}\right)$ is the displacement (in direction $(i)$ ) produced by a localized impulse force (in direction $(m))$ in space $\left(x^{\prime}\right)$ and time $\left(t^{\prime}\right)$ :

$$
\rho \frac{\partial^{2} G_{i m}}{\partial t^{2}}\left(x, x^{\prime} ; t-t^{\prime}\right)-\frac{\partial}{\partial x_{j}} \sigma_{i j}^{G m}\left(x, x^{\prime} ; t-t^{\prime}\right)=\delta_{i m} \delta\left(x-x^{\prime}\right) \delta\left(t-t^{\prime}\right),
$$

and satisfies that the normal stresses it produces at the free surface of the sample are zero. 


\section{Stress fields due to dislocation loops in finite samples}

\subsection{Stationary case}

Starting from Eq. (1D), the stress field produced by the static dislocation loop is:

$$
\sigma_{p q}(x)=-b_{i} C_{p q l m} \int_{S^{\prime}} d S_{j}^{\prime} \frac{\partial}{\partial x_{l}} \sigma_{i j}^{G m}\left(x^{\prime}, x\right) .
$$

This can be expressed as a line integral along the dislocation loop:

$$
\sigma_{p q}(x)=b_{i} C_{p q l m} \int_{L^{\prime}} d l_{s}^{\prime}\left\{\epsilon_{s j l} \sigma_{i j}^{G m}\left(x^{\prime}, x\right)-A_{s}^{i l m}\left(x^{\prime}, x\right)\right\},
$$

with the "vector potential" $A_{s}^{i l m}\left(x^{\prime}, x\right)$ to be determined for each specific sample. It is defined by:

$$
\frac{\partial}{\partial x_{l}} \sigma_{i j}^{G m}\left(x^{\prime}, x\right)+\frac{\partial}{\partial x_{l}^{\prime}} \sigma_{i j}^{G m}\left(x^{\prime}, x\right)=\epsilon_{j r s} \frac{\partial}{\partial x_{r}^{\prime}} A_{s}^{i l m}\left(x^{\prime}, x\right) .
$$

\subsection{Non stationary case}

Since the problem is homogeneous under time translations it is possible to obtain an expression for particle velocity, that involves an integral along the dislocation loop only. Indeed, from Eq. (1):

$$
\frac{\partial U_{m}}{\partial t}(x, t)=b_{i} \int_{-\infty}^{\infty} d t^{\prime}\left\{\frac{\partial}{\partial t^{\prime}}\left[\int_{S\left(t^{\prime}\right)} d S_{j}^{\prime} \sigma_{i j}^{G m}\left(x^{\prime}, x ; t-t^{\prime}\right)\right]-\int_{\frac{d S}{d t^{\prime}}\left(t^{\prime}\right)} d S_{j}^{\prime} \sigma_{i j}^{G m}\left(x^{\prime}, x ; t-t^{\prime}\right)\right\} .
$$

The first term is zero since the Green's function vanishes at $t-t^{\prime}= \pm \infty$. Since $\int_{d S\left(t^{\prime}\right) / d t^{\prime}} d S_{j}^{\prime}=$ $\epsilon_{j p q} \int_{L\left(t^{\prime}\right)} d l_{q}^{\prime} V_{p}\left(x^{\prime}, t^{\prime}\right)\left(L\left(t^{\prime}\right)\right.$ is the dislocation loop bounding the slip plane $S\left(t^{\prime}\right), V_{p}\left(x^{\prime}, t^{\prime}\right)$ is the loop's local velocity and $\epsilon_{j p q}$ the completely antisymmetric tensor in three dimensions), we have:

$$
\frac{\partial U_{m}}{\partial t}(x, t)=-b_{i} \int_{-\infty}^{\infty} d t^{\prime} \int_{L\left(t^{\prime}\right)} d l_{q}^{\prime} \sigma_{i j}^{G m}\left(x^{\prime}, x ; t-t^{\prime}\right) \epsilon_{j p q} V_{p}\left(x^{\prime}, t^{\prime}\right) .
$$

From this expression for the displacement velocity field, one obtains an expression for the partial time derivative of the stress field that involves line integrals over the dislocation loop:

$$
\frac{\partial}{\partial t} \sigma_{p q}(x, t)=-C_{p q k m} b_{i} \int d t^{\prime} \int_{L\left(t^{\prime}\right)} d l_{q}^{\prime} \frac{\partial}{\partial x_{k}} \sigma_{i j}^{G m}\left(x^{\prime}, x ; t-t^{\prime}\right) \epsilon_{j p q} V_{p}\left(x^{\prime}, t^{\prime}\right) .
$$

As mentioned in the Introduction, this result together with the result for a static loop (considered as an initial condition) mean that the time dependent stress field can be written in terms of integration of line integrals over the dislocation loop, consequently it is independent of the choice of slip planes.

\section{Examples for the stationary case}




\subsection{A screw dislocation in a half space}

Using the method in Eshelby and Stroh (1951) the displacement and stress fields in polar coordinates due to a screw dislocation lying along the $z$ axis perpendicular to the free surface of a half space, can be obtained as (the half space exists for $z>0$ ):

$$
\begin{aligned}
U_{z}(\theta) & =\frac{b}{2 \pi} \theta \\
U_{\theta}(r, z) & =\frac{b}{2 \pi} \int_{0}^{\infty} \frac{d k}{k} e^{-k z} J_{1}(k r) \\
\sigma_{z \theta}(r, z) & =\frac{\mu b}{2 \pi r}-\frac{\mu b}{2 \pi} \int_{0}^{\infty} d k e^{-k z} J_{1}(k r) \\
\sigma_{r \theta}(r, z) & =-\frac{\mu b}{2 \pi} \int_{0}^{\infty} d k e^{-k z} J_{2}(k r)
\end{aligned}
$$

( $J_{1}(z)$ the Bessel's function of order one). Applying the general formula of Eq. (7):

$$
\sigma_{z \eta}(\vec{R}, z)=b \mu \epsilon_{\alpha \eta} \int_{0}^{\infty} d z^{\prime} \sigma_{z \alpha}^{G z}\left(z^{\prime}, z ;-\vec{R}\right)-b \mu \int_{C} d R_{\beta}^{\prime} A_{\beta}^{z z \delta}\left(z^{\prime}=0, z ; \vec{R}^{\prime}-\vec{R}\right)
$$

with greek indices indicating coordinates $x$ or $y$ only, $\vec{R} \equiv x \hat{i}+y \hat{j}, \mu$ the shear elastic constant, and $C$ is an arbitrary curve on the free surface. The "vector potential" $A_{\beta}^{z z \delta}$ at the free surface is given in Fourier space by:

$$
A_{\beta}^{z z \delta}\left(z^{\prime}=0, z ; \vec{k}\right)=\frac{e^{-k z}}{k^{2}} k_{\beta} \epsilon_{\delta \eta} k_{\eta} .
$$

If one calculates $\sigma_{z \theta}(r, z)$ using Eq. (13), one reproduces the first and second terms in Eq. (12) with the equivalent terms in Eq. (13). Gosling and Willis (1994) have given a different line integral representation specifically determined for the geometry of an isotropic half space.

\subsection{A screw dislocation in a plate}

In Eshelby et al. (1951) the exact displacement and stress fields in polar coordinates due to a screw dislocation along the $z$ axis perpendicular to the free surfaces of a plate, were obtained as (the plate exists for $-h<z<h$ ):

$$
\begin{aligned}
U_{z}(\theta) & =\frac{b}{2 \pi} \theta \\
U_{\theta}(r, z) & =-\frac{b}{2 \pi} \int_{0}^{\infty} \frac{d k}{k} \frac{\sinh (k z)}{\cosh (k h)} J_{1}(k r) \\
\sigma_{z \theta}(r, z) & =\frac{\mu b}{2 \pi r}-\frac{\mu b}{2 \pi} \int_{0}^{\infty} d k \frac{\cosh (k z)}{\cosh (k h)} J_{1}(k r) \\
\sigma_{r \theta}(r, z) & =\frac{\mu b}{2 \pi} \int_{0}^{\infty} d k \frac{\sinh (k z)}{\cosh (k z)} J_{2}(k r) .
\end{aligned}
$$

The calculation of $\sigma_{z \theta}(r, z)$ using Eq. (7) is analogous to the half-space case. One needs the "vector potential" $A_{\beta}^{z z \delta}$ evaluated at the surface, which in Fourier space is:

$$
A_{\beta}^{z z \delta}( \pm h, z ; \vec{k})=-\frac{k_{\beta} \epsilon_{\delta \nu} k_{\nu}}{2 k^{2}}\left\{ \pm \frac{\cosh (k z)}{\cosh (k h)}+\frac{\sinh (k z)}{\sinh (k h)}\right\}
$$




\section{Acknowledgments}

This work was supported in part by the Andes Foundation, Fondecyt Grant 3950011 and a Cátedra Presidencial en Ciencias. Useful discussions with F. Lund are gratefully acknowledged. 


\section{References}

- Boudet, J.F., Ciliberto S., and Steinberg J., J. Phys. II France, 6, 1493 (1996).

- Eshelby, J.D., and Stroh, A.N., Philos. Mag., Ser. 7, Vol. 42, 1401 (1951).

- Gosling, T.J., and Willis, J.R., J. Mech. Phys. Solids, Vol. 42, no 8, 1199 (1994).

- Lund, F., Phys. Rev. Lett., 76, 2742 (1996).

- Mura, T., Philos. Mag. 8, 843 (1963).

- Sharon, E., Gross, S.P, and Fineberg, J., Phys. Rev. Lett., 76, 2117 (1996). 